\title{
Leiomyosarcoma of the spermatic cord: case report and literature review
}

\author{
Pankaj Dangle, MS, MRCS, MCh;* \\ Doddametikurke R. Basavaraj, MS, FRCS;* \\ Selina Bhattarai, MRCPath; ${ }^{\dagger}$ Alan B. Paul, MBChB, FRCSEd; ${ }^{\neq}$ \\ Chandra Shekhar Biyani, MS, D Urology, FRCS, FEBU*
}

\begin{abstract}
A 51-year-old man presented with a 2-year history of painless lump in the left hemiscrotum. Scrotal examination demonstrated a 3-cm, firm-to-hard mass attached to the left spermatic cord. Scrotal ultrasound scan revealed a heterogeneous mass separate from the left testis and epididymis. An orchidectomy was recommended to the patient; however, he declined surgery and underwent excision biopsy of the lesion with preservation of the left testis. Histology suggested a leiomyosarcoma of the spermatic cord and positive margins. Following a normal staging CT scan, the patient was referred to the regional sarcoma clinic. He was treated with an orchidectomy. Microscopic examinations showed fibrosis in the spermatic cord and negative margins. The patient has been followed up for 12 months with CT scans and shows no signs of recurrence.
\end{abstract}

CUAJ 2007; 1(1):55-8

\section{Case Report}

A 51-year-old man presented with a 2-year history of painless lump in the left hemiscrotum. He had no history of trauma, sudden increase in size or associated pain. The patient denied any lower urinary tract symptoms. His previous medical history included recurrent renal stone disease. Clinical examination of the scrotum revealed a $3 \mathrm{~cm}$, non-tender, firm-to-hard lump at the root of the scrotum separate from the left testis. The lump was not freely mobile and was attached to the left spermatic cord. The routine blood investigations were normal. Ultrasound scan of the scrotum showed normal appearance on the right side, with a distinct and well circumscribed $4 \times 3.2 \times 2.5 \mathrm{~cm}$ lesion of mixed heterogeneous echogenicity on the left side. It was abutting the upper pole of the left epididymal head but separate from the epididymis and the testis. The lesion itself showed increased vascularity (Fig. 1A and Fig. 1B).

The patient was offered a radical orchidectomy along with excision of the spermatic cord mass, but he declined to have an orchidectomy and opted only for excision of the mass. Upon operating we discovered that he had a lump arising from the cord near the root of the left hemiscrotum.
The testis and epididymis were separate from the lesion. The lump was dissected from the cord structure. The cut surface was white, solid and hard to the touch. The testis was repositioned in the scrotum and the inguinal incision was closed in layers. The patient's postoperative recovery was uneventful.

Microscopic examination revealed cells arranged in well defined fascicles with central hyperchromatic nuclei and a moderate amount of eosinophilic cytoplasm (Fig. 2). There were 4 mitoses per 10 high power fields. Small foci of necrosis were noted with the appearances of a spindle cell lesion of probable smooth muscle origin. The high cellularity, pleomorphism, necrosis and mitotic activity suggested this was a malignant tumour. The tumour was classified as a grade 2 (differentiation $=2$, mitoses $=1$, necrosis $=1$ ) on the Fédération Nationale des Centres de Lutte Contre le Cancer system. These histological features were suggestive of a leiomyosarcoma. The immunohistochemistry revealed interlacing fascicles of smooth muscle (positive for desmin, smooth actin and negative for $S$ 100) (Fig. 3A and Fig. 3B). The histology showed infiltration at edges with margins positive for tumour.

The diagnosis of leiomyosarcoma and positive margin on histology prompted us to refer the patient to the regional sarcoma clinic for further treatment. His staging CT san of the chest, abdomen and pelvis did not reveal any local or distant tumour. Subsequently, he underwent a left radical orchidectomy with histology showing fibrosis of the spermatic 
cord and negative margin. Clinical and radiologic follow-up with CT of the chest, abdomen and pelvis at 12 months showed no recurrence.

\section{Discussion}

Malignant neoplasms of non-testicular origin are uncommon and are generally sarcomas. In a series of 1583 adult soft tissue sarcomas at the Memorial Sloan-Kettering Cancer Center, 43 were urological and 14 $(0.8 \%)$ were paratesticular (5 rhaddomyosarcomas, 4 leiomyosarcoma, 3 liposarcomas, 1 malignant fibrous histiocytoma and 1 undifferentiated sarcoma). ${ }^{1}$

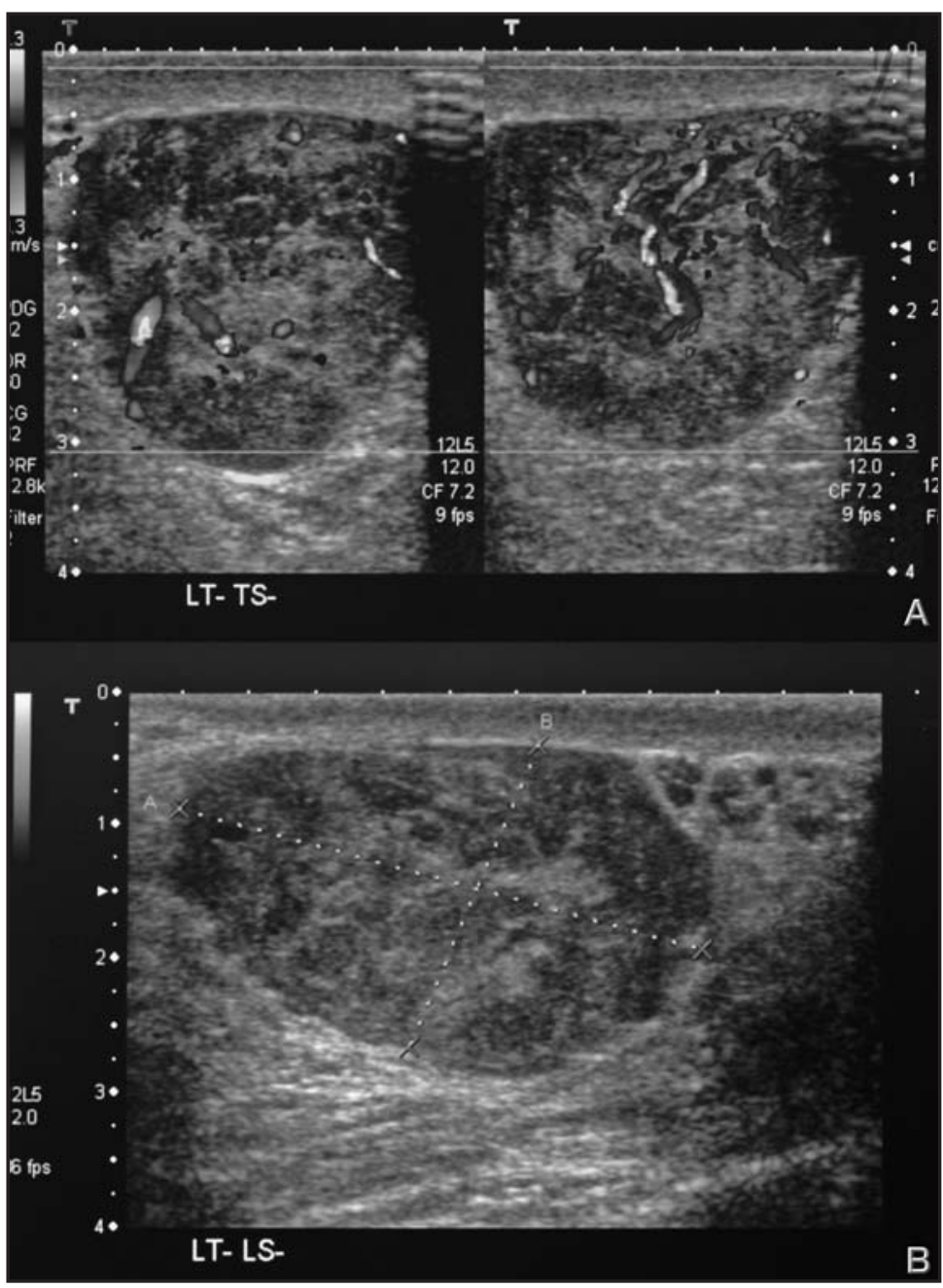

Fig. 1. Fig. $1 \mathrm{~A}$ is a scrotal ultrasound showing a well-circumscribed lesion of mixed heterogenous echogenicity on the left side. Fig. 1B is a Doppler scan demonstrating increased vascularity.
Leiomyosarcoma accounts for $5 \%-10 \%$ of soft tissue sarcoma. ${ }^{2}$ Though leiomyosarcoma of the spermatic cord is rare, about 110 cases have been reported in the literature. ${ }^{2}$ A review of 10 series of paratesticular sarcomas in adults showed that leiomyosarcoma is the most commonly reported histological variety, with a peak incidence in the sixth and seventh decade. ${ }^{2}$ Leiomyosarcoma originates from the spermatic cord, the scrotum or the epididymis. The most common spermatic cord type arises from undifferentiated mesenchymal cells of the cremasteric muscle and vas deferens. The less frequent epididymal form originates from the smooth muscle surrounding the basement membrane of the epididymis canal. The scrotal types are described as arising from the dartous layer. The spermatic and epididymal types drain to the retroperitoneal lymph nodes in contrast with the scrotal form that drains to the inguinal, internal and external iliac nodes.

Due to the rare occurrence of the disease, it is important to understand the modes of spread. The most common means of spread is lymphatic, then hematogenous and last, by local extension. The route of lymphatic dissemination may involve the external iliac, hypogastric, common iliac and para aortic nodes. The lung is the primary site for blood-borne metastases. Local spread to the scrotum, inguinal canal or pelvis is possible along the pathway of the vas deferens.

The natural course of leiomyosarcoma depends on site, size, grade and evidence of nodal or distant metastasis. Anatomically, leiomyosarcoma is divided into 3 subgroups: deep soft tissue, cutaneous-subcutaneous and vascular origin. According to the American Joint Committee on Cancer, staging system spermatic cord leiomyosarcoma is a deeper variety of subgroup. ${ }^{3}$

Preoperative diagnosis of spermatic cord leiomyosarcoma is difficult and usually made by histological examination. Clinically leiomyosarcoma presents as a 
painless, firm, para-testicular intrascrotal mass and therefore diagnostic evaluation should be similar too that of any testicular tumour. Scrotal ultrasound is a useful primary way to assess the mass and its relation to the testis. Once the diagnosis of leiomyosarcoma has been established by surgery, clinical staging is necessary. Magnetic resonance imaging can be a useful problem-solving tool and is particularly helpful in better characterizing extra testicular solid masses, as well as delineating the anatomic extent of the tumour. ${ }^{4}$

Radical orchidectomy is the standard primary surgical procedure followed by adjuvant radiotherapy to reduce the local recurrence. The primary treatment with radical orchidectomy is an essential approach, but a single case report with local excision and surveillance has been reported. ${ }^{5}$ A reported survival rate is $50 \%-80 \%$ and it has been observed that simple excision is suboptimal as repeat wide excision has demonstrated microscopic residual disease in $27 \%$ of cases and therefore warrants an additional adjuvant treatment. ${ }^{6} \mathrm{~A}$ wide excision is almost mandatory if negative histological margins are not achieved during primary surgery. Even if extensive resection is required to clear the margins, advances in miscrosurgical techniques have made it possible to reconstruct large anatomic defects in this area by local flaps. ${ }^{7}$ Due to the high incidence of loco-regional recurrence in the lymph nodes, 2 different treatment alternatives, prophylactic retroperitoneal lymph node dissection (RPLND) and radiotherapy, have been tried. The proponents of RPLND indicate that there is a $29 \%$ risk of metastatic potential to regional lymph nodes. A review of 101 patients by Banowsky and Schultz described 29 cases of RPLND with lymph node involvement. Of these, 17 patients had isolated lymphatic dissemination. Despite such a high incidence of lymphatic spread, no report has yet shown a significant survival benefit from the addition of RPLND to radical orchidectomy. ${ }^{8}$
A study from Massachusetts involved 18 patients with spermatic cord sarcoma who were subdivided into 2 groups of 9 patients. One group had surgery and the other group had surgery plus radiation. Of the 9 patients treated with radical orchidectomy alone, 5 developed loco-regional failure, 2 of which were limited to lymph nodes. In contrast, there were no loco-regional recurrences among the 9 patients who received adjuvant radiation to the regional lymph nodes. ${ }^{9}$ These findings, which are consistent with those of Catton and colleagues, suggest that adjuvant radiation may effectively control loco-regional microscopic disease..$^{10}$ In our case, histology from the orchidectomy (the specimen) showed clear margins, therefore, radiotherapy was not recommended.

In conclusion, spermatic cord leiomyosarcoma, although rare, should be one of the first differential diagnoses for a firm-to-hard lump in the cord. Apart from radical orchidectomy, there has been added benefit of adjuvant radiotherapy to prevent any loco-regional lymph node recurrence.

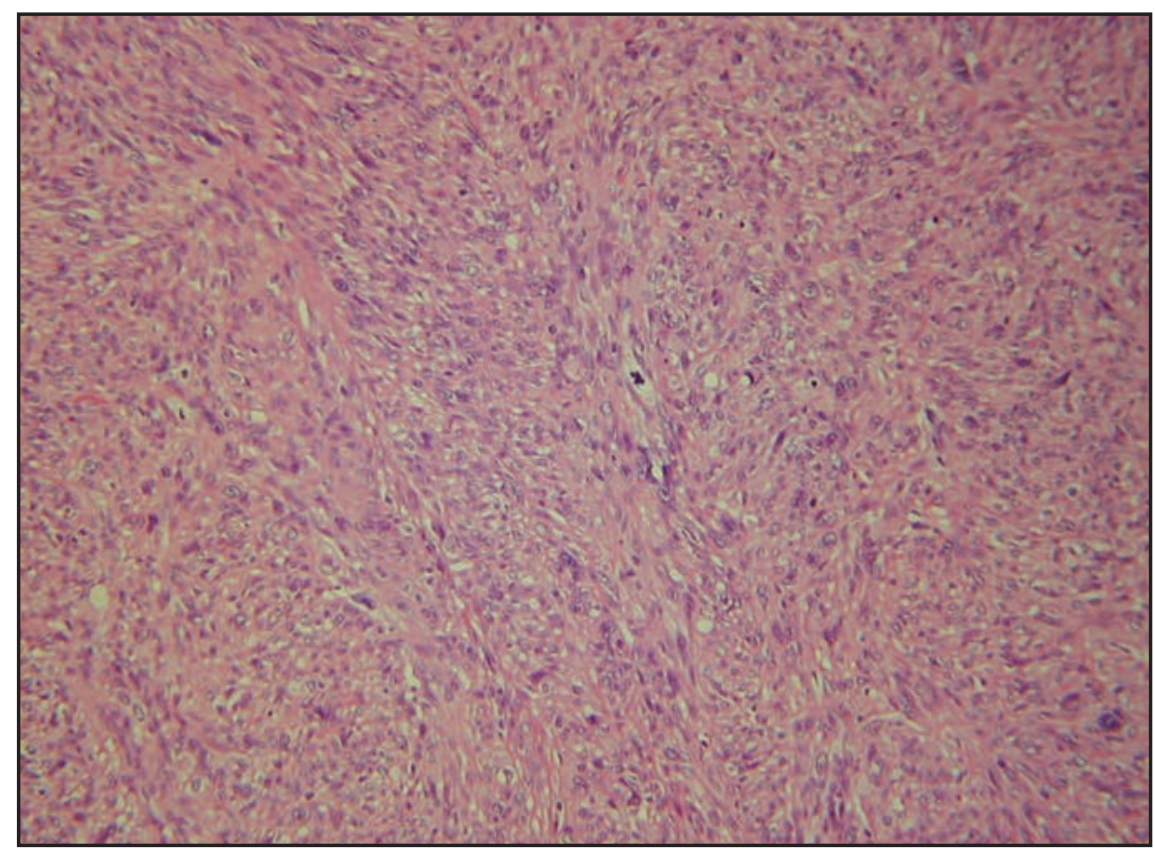

Fig. 2. Interlacing fascicles of spindle cells showing nuclear pleomorphism and abnormal mitosis; hematoxylene and eosin staining at 200X magnification. 


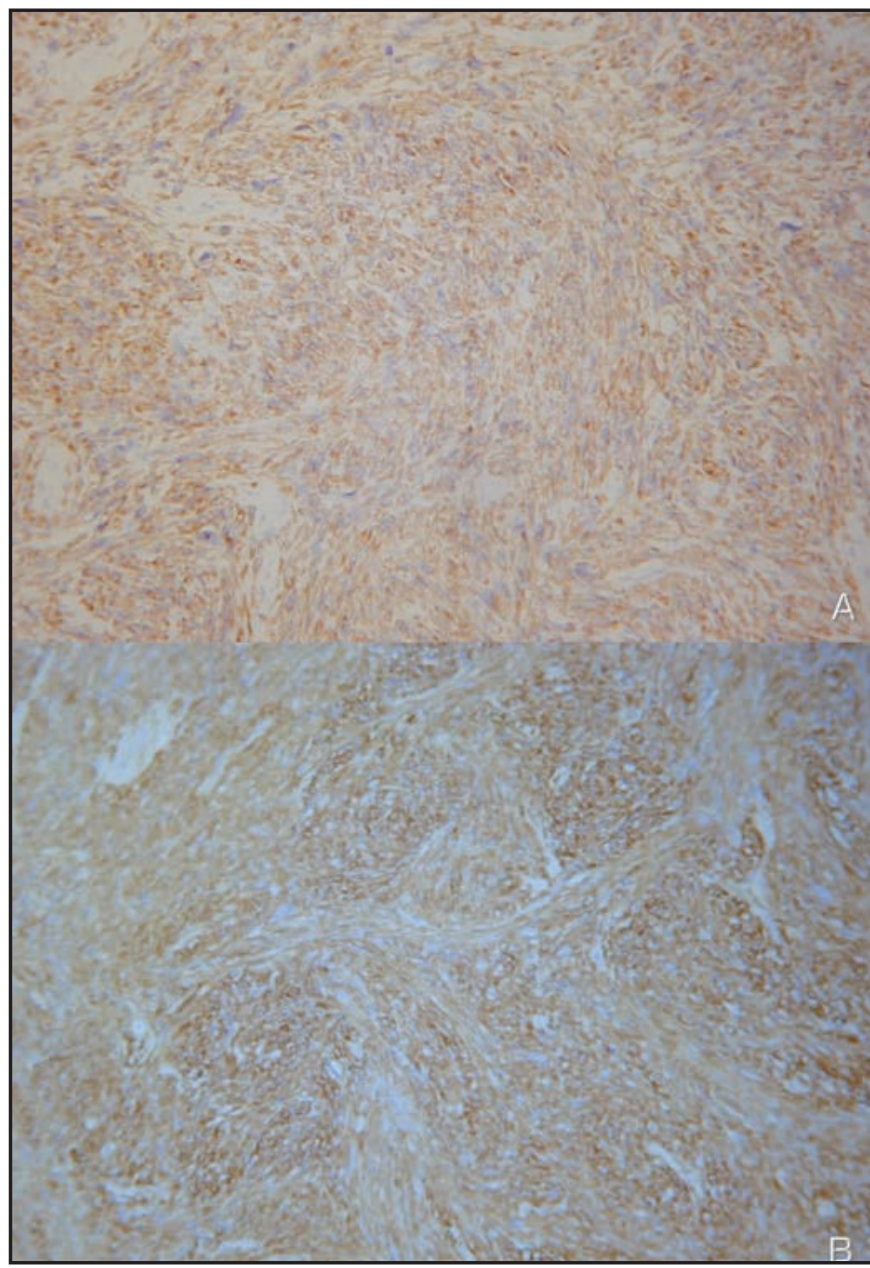

Fig. 3. Tumor cells showing positive staining for desmin (Fig. 3A) and smooth muscle actin (Fig. 3B) at 200X magnification.
From the *Department of Urology, Pinderfields General Hospital, Wakefield, UK, the †Department of Urology and the ‡Department of Pathology, St James's University Hospital, Leeds, UK

This article has been peer reviewed.

Competing interests: None declared.

\section{References}

1. Russo P. Urologic sarcoma in adults. Urol Clin North Am 1991;18:581-7.

2. Weiss SW, Goldblum JR. Leiomyosarcoma, In: Enzinger and Weiss's Soft tissue Tumors. 4th ed. St.Louis (MI): Mosby Inc; 2001. p. 727-48.

3. Folpe AL, Weiss SW. Paratesticular soft tissue neoplasms. Semin Diagn Pathol 2000;17:307-18.

4. Woodward PJ, Schwab CM, Sesterhenn IA. Extra testicular scrotal masses. Radiographics 2003;23:215-40.

5. Lopes RI, Leite KR, Lopes RN. Paratesticular leiomyosarcoma treated by enucleation. Int Braz J Urol 2006;32:66-7.

6. Blitzer PH, Dosoretz DE, Proppe KH, et al. Treatment of malignant tumors of the spermatic cord: a study of 10 cases and a review of the literature. J Urol 1981;126:611-4.

7. Enoch S, Wharton SM, Murray DS. Management of leiomyosarcoma of the spermatic cord: the role of reconstructive surgery. World I Surg Oncol. 2005;28;3:23.

8. Banowsky LH, Schultz GN. Sarcoma of spermatic cord and tunics: review of literature, case report and discussion of role of retroperitoneal lymph node dissection. J Urol 1970;103:628-31.

9. Fagundes MA, Zietman AL, Althausen AF, et al. The management of spermatic cord sarcoma. Cancer 1996;77:1873-6.

10. Catton CN, Cummings BJ, Fornasier V, et al. Adult paratesticular sarcomas: a review of 21 cases. J Urol 1991;146:342-5.

Correspondence: Mr. Chandra Shekhar Biyani, Department of Urology, Pinderfields General Hospital, Mid Yorkshire Hospitals NHS Trust, Aberford Road, Wakefield, West Yorkshire, UK WFI 4DG; shekharbiyani@hotmail.com 